\title{
Adult female with symptomatic AVPR2-related nephrogenic syndrome of inappropriate antidiuresis (NSIAD)
} \author{
Mohamed Majeed ${ }^{2}$, Mark Gurnell ${ }^{2}$, Soo-Mi Park ${ }^{1}$ and Helen Simpson ${ }^{5}$ \\ Departments of ${ }^{1} \mathrm{Clinical}$ Genetics, ${ }^{2}$ Diabetes and Endocrinology, Cambridge University Hospitals NHS Foundation \\ Trust, Cambridge, UK, ${ }^{3}$ Department of Medical Genetics, University of Cambridge and NIHR Cambridge Biomedical \\ Research Centre and Cancer Research UK Cambridge Centre, Cambridge, UK, ${ }^{4}$ Department of Clinical Biochemistry, \\ Cambridge University Hospitals NHS Foundation Trust, Cambridge, UK, and ${ }^{5}$ Department of Diabetes and \\ Endocrinology, UCLH NHS Foundation Trust, London, UK
}

Jennifer Hague1, Ruth Casey2,3, Jonathan Bruty', Tom Legerton', Stephen Abbs', Susan Oddy4, Andrew S Powlson²,

\section{Correspondence} should be addressed to J Hague

Email

jennifer.hague@ addenbrookes.nhs.uk

\section{Summary}

Activating mutations in AVPR2 are associated with nephrogenic syndrome of inappropriate antidiuresis (NSIAD). NSIAD causes hyponatremia, decreased serum osmolality and clinical symptoms, which may present from birth or in infancy and include hypotonia, irritability, vomiting and/or seizures. Symptoms in later life are often less specific and include malaise, dizziness, confusion, tiredness and headache. NSIAD is a rare X-linked condition, which is associated with a variable phenotype in males, of whom some present in infancy but others do not become symptomatic until adulthood, or occasionally, never. Female carriers may present with episodes of hyponatremia, usually found incidentally. Literature in this field is limited; namely, two clinical reports describing a female proband, both diagnosed in infancy. We describe, for the first time, the case of an adult female proband with NSIAD, who had longstanding associated symptoms of tiredness, headache, temporary memory loss and mood changes as well as hyponatremia and decreased serum osmolality. A water load test demonstrated an inability to dilute urine and gene sequencing confirmed a recurrent activating mutation in AVPR2. The variant was inherited from the proband's mother who had had longstanding episodes of transient asymptomatic hyponatremia. This is the third report of a female proband with NSIAD and is the first female reported who sought medical treatment for chronic symptoms from adulthood. This case acts as a reminder of the importance of considering NSIAD as a diagnosis in females of all ages with unexplained hyponatremia.

\section{Learning points:}

- Activating mutations in the AVPR2 gene are associated with the rare X-linked condition nephrogenic syndrome of inappropriate antidiuresis.

- NSIAD is associated with hyponatremia, decreased serum osmolality and inappropriately increased urinary osmolality. Early clinical symptoms in infancy include hypotonia, irritability, vomiting and/or seizures. Symptoms in later life include malaise, dizziness, confusion, tiredness and headache.

- NSIAD should be considered in female, as well as male, patients who present with unexplained hyponatremia and decreased serum osmolality. Family history may reveal relevant symptoms or biochemical features in other family members. However, family history may not always be informative due to the variable nature of the condition or if the proband has a de novo pathogenic variant. 
- A water load test with measurement of AVP may be informative in distinguishing NSIAD from SIADH. Measurement of co-peptin levels may be considered, in substitution for direct measurement of AVP.

- Patients with NSIAD should be counseled about appropriate daily fluid volume intake. Potential episodes of fluid overload should be avoided.

\section{Background}

Nephrogenic syndrome of inappropriate antidiuresis (NSIAD, OMIM 300539) is associated with gain-offunction pathogenic variants in AVPR2, which encodes the vasopressin V2 receptor (1). Activating mutations cause constitutive activation of the vasopressin receptor resulting in antidiuresis and therefore cause the biochemical and clinical features of NSIAD. In contrast, loss-of-function mutations in AVPR2 cause X-linked congenital nephrogenic diabetes insipidus (2).

NSIAD has been traditionally described as an X-linked recessive condition with index cases usually being male but with marked interfamilial and intrafamilial heterogeneity regarding the age of diagnosis and severity of disorder. To date, carrier females have most commonly been asymptomatic and identified during familial cascade screening. The two exceptions have been clinical reports of females who were both diagnosed with NSIAD in infancy $(3,4)$. However, a female proband presenting with chronic symptoms in adulthood has not been previously described until now. We describe the clinical features of our patient and describe relevant investigations and management. We outline the diagnostic utility of a water load test for NSIAD prior to molecular sequencing of AVPR2. Furthermore, we demonstrate that measurement of co-peptin levels can be used as an alternative to direct AVP measurement during the water load test.

\section{Case presentation}

Our patient was an only child and was born to nonconsanguineous white British parents. Her mother had had difficulty conceiving, possibly due to a congenital uterine malformation and underwent hysterectomy after our patient was born. The proband was born at term after a normal pregnancy. Her development and early medical history were unremarkable. She had glandular fever at the age of 16 years and described feeling continually unwell from that time. Between the ages of 16 and 30 years she regularly experienced symptoms of nausea, headache and malaise, which were significant enough for her to attend the local hospital emergency room. On these occasions, she was found to be hyponatremic (serum sodium around $125 \mathrm{mmol} / \mathrm{L}$ ) with normal serum potassium and bicarbonate levels. Clinical management included hospital admission and fluid restriction. This resulted in normalization of her serum sodium levels and resolution of her clinical symptoms. She also reported other symptoms, related to the episodes of hyponatremia, and these included tiredness, occasional memory loss, mood changes and depressive symptoms. There was no history of seizures.

In her twenties, she was given a provisional diagnosis of SIADH based on the biochemical profile of persistent hyponatremia with plasma serum sodium of $125-132 \mathrm{mmol} / \mathrm{L}$ (reference range 133-146 $\mathrm{mmol} / \mathrm{L}$ ) associated with a low serum osmolality of around $270 \mathrm{mosmol} / \mathrm{kg}$ (reference range 275-295 mosmol/kg) while euvolemic. Management included fluid restriction and sodium chloride supplements, which had variable effect, possibly due to tolerability. Due to lack of consistent improvement, she underwent a trial of demeclocycline, but again with limited sustained benefit.

Additionally aged 16 years, she was investigated for primary ovarian insufficiency but no cause was identified. Genetic testing failed to identify either a Fragile X syndrome premutation or any chromosomal abnormalities. She also underwent investigations for autoimmune disorders, which were negative. The premature ovarian failure resulted in sub-fertility, and she later adopted two children. She underwent investigations for chronic fatigue syndrome and was diagnosed with fibromyalgia.

\section{Investigation}

She was further investigated as no underlying cause had been found for her symptoms and biochemical profile. At this time, she remained euvolemic, with a plasma sodium of $133-136 \mathrm{mmol} / \mathrm{L}$, but urine osmolality was raised (626 mosmol/kg). Adrenocortical and thyroid function 


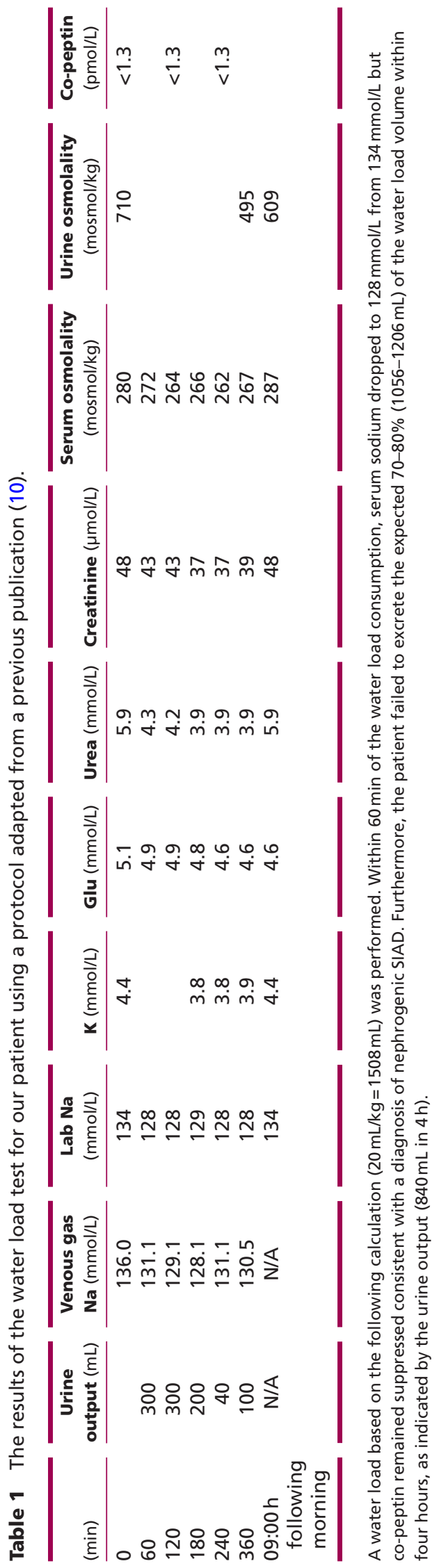

tests and an acute intermittent porphyria screen were normal. She was taking no medication which could be responsible for her symptoms. Cross-sectional imaging of the head, chest and abdomen did not identify an infectious or neoplastic cause for SIADH and an octreotide isotope scan was also normal.

A water load test was therefore considered to determine if the hyponatremia was due to an inability to dilute urine in the presence of a relative excess of free water (Table 1). This test was carried out in a controlled environment and under direct supervision, given the risk of exacerbating hyponatremia associated with this test. This investigation demonstrated a marked inability of the patient to excrete a free water load $(40 \%$ of a $20 \mathrm{~mL} / \mathrm{kg}$ oral fluid load by 240 min post ingestion). Normal subjects excrete $78-82 \%$ of the water load in $4 \mathrm{~h}$. There was onset of hyponatremia at $128 \mathrm{mmol} / \mathrm{L}$ and a persistently elevated urine osmolality during the test. However, serum co-peptin, a peptide released together with AVP, was undetectable throughout the test $(<1.3 \mathrm{pmol} / \mathrm{L})$, which indicated that our patient did not have SIADH. The water load test was therefore important in establishing that NSIAD was the most likely diagnosis in our patient. The value of the water load test in the diagnosis of NSIAD is described by Ranchin and coworkers (5).

To confirm the diagnosis, next-generation sequencing (NGS) was carried out with the Illumina TruSight One sequencing panel using the MiSeq Illumina NGS platform. Reads were aligned; variants were called and initial prefiltering was carried out using an in-house bioinformatics pipeline. This pipeline filtered the data to only show AVPR2 variants. Variants with a population frequency greater than 1 in 50 were automatically deemed as insignificant.

Variant interpretation of the remaining variants was carried out using the Alamut Visual software. All variants of class 4 or above, according to the current ACMG guidelines, were then confirmed by Sanger sequencing (6). The recurrent mutation c.409C > T, p.(Arg137 Cys) was identified in AVPR2, confirming the diagnosis of NSIAD. $\mathrm{X}$-linked inactivation studies were requested and showed an unskewed pattern in blood.

The pathogenic variant was found to be maternally inherited. On review of the medical records, our patient's mother had experienced recurrent episodes of hyponatremia during illness, for example, after an episode of shingles, but also on routine medical review, with the lowest serum sodium recorded at $130 \mathrm{mmol} / \mathrm{L}$. She also had a history of lupus and Hashimoto's thyroiditis but did not display any symptoms of NSIAD. Her parents were 
deceased, and her two brothers (one deceased) did not have any relevant symptoms. However, predictive genetic testing has been offered to the wider family.

\section{Treatment}

Our patient is now asymptomatic on daily fluid restriction of 1-1.5 L. This recommended volume of fluid was based on the lowest daily fluid volume our patient felt she could tolerate, while ensuring that her sodium levels remain just under or within the normal range (usually between 132 and $135 \mathrm{mmol} / \mathrm{L})$.

\section{Outcome and follow-up}

Our patient has been followed up for a further 12 months, since she received her genetic diagnosis. She remains asymptomatic on fluid restriction.

\section{Discussion}

NSIAD was first described in 2005 in two infant boys (1). Since then, around 30 cases have been reported. Several activating mutations have been described but our patient's mutation, R137C, causing an arginine to cysteine change, is the most commonly reported to date.

NSIAD is inherited in an X-linked manner and the majority of index patients have been males. However, there have been several reports where females with NSIAD have been identified on cascade screening $(3,7$, 8). Asymptomatic carrier females have been reported who have normal plasma sodium and osmolality (1). In contrast, other asymptomatic female carriers have had documented episodes of hyponatremia and/or when investigated with a water load test, have had impaired ability to dilute urine $(3,7,8)$. Decaux and coworkers described a female who had molecularly-confirmed NSIAD but did not have episodes of hyponatremia or an abnormal water load test. X-inactivation studies were compatible with preferential inactivation of her mutated allele (7). Gupta and coworkers reported a male infant and his mother who both had NSIAD. The mother had learned to avoid excess fluid consumption because of associated malaise. Both family members had a subnormal ability to excrete a water load, with the mother also demonstrating a heightened sense of thirst at low serum osmolalities (8). Ranchin and coworkers described the value of the water load test in helping to diagnose NSIAD and distinguish it from cases of SIADH. The paper describes the results of the water load test that would be expected in both hemizygous males and heterozygous females with an activating AVPR2 pathogenic variant (5).

There have been two reports of female probands who both presented in infancy $(3,4)$. A 1-year-old girl was referred for persistent hyponatremia, which was first noted incidentally during an intercurrent infection. She had global developmental delay, although it is unclear if this was related to her diagnosis of NSIAD. Her mother reported a strong aversion to fluids, because she subsequently would experience headaches, which could be relieved only by eating salty foods. She passed urine only once or twice a day. The maternal grandmother reported similar symptoms. Genetic studies confirmed NSIAD in all family members (3). In the second case, the infant presented with diarrhea and poor feeding at 23 days of age. She was found to have mild hypertension and continuing episodes of hyponatremia. Her brother was known to have NSIAD and the diagnosis was also confirmed in her, with chromosome inactivation studies showing an unskewed X-inactivation pattern (4).

Identifying patients with NSIAD is important for symptom control as well as long-term management of fluid restriction. It is especially important to consider the diagnosis in infancy if a child presents with irritability, vomiting and/or seizures and a biochemical picture of SIADH, as SIADH is rare in pediatric patients and NSIAD is more likely, especially if the patient has low or undetectable AVP levels. Immediate management includes fluid restriction and possibly medication, including the osmotic agent urea and inverse agonists for specific variants, e.g. p.(Phe299Val) and p.(lle130Asn) $(9,10)$. It is important to counsel patients on appropriate daily fluid volume intake and to ensure that episodes of fluid overload can be avoided, such as increased fluid intake in summertime or during sports activities or excessive intravenous fluid administration during hospital admissions.

In conclusion, we propose that NSIAD should be considered as a diagnosis in female, as well as male, patients who present with unexplained hyponatremia and decreased serum osmolality. A water load test may be informative, and measurement of co-peptin levels may be considered, in substitution for direct measurement of AVP, to aid diagnosis and guide diagnostic sequencing of AVPR2. In families with a known history of NSIAD, we would recommend that both females and males, from birth onwards, should be offered genetic testing for the familial variant and, if positive, referred to endocrinology for long-term management. Due to the increasing availability of genetic testing, confirmation of diagnosis is becoming easier. We conclude that, as with many other 
$\mathrm{X}$-linked disorders, the concept of a truly recessive X-linked inheritance is becoming blurred. This is explained by the random and mosaic nature of $\mathrm{X}$-inactivation in females.

\section{Declaration of interest}

The authors of this clinical report declare that there is no conflict of interest that could be perceived as prejudicing the impartiality of the research reported.

\section{Funding}

This research did not receive any specific grant from any funding agency in the public, commercial or not-for-profit sector.

\section{Patient consent}

We express our gratitude to our patient for her written consent for the publication of this case report.

\section{Author contribution statement}

Jennifer Hague wrote the manuscript. Ruth Casey wrote Table 1 and reviewed the paper. Andrew S Powlson, Mohamed Majeed and Mark Gurnell were the patient's endocrine physicians and reviewed the paper. Tom Legerton, Jonathan Bruty and Stephen Abbs performed and interpreted the molecular sequencing and $\mathrm{X}$-inactivation studies. Susan Oddy performed the biochemical analysis. Soo-Mi Park was the patient's clinical genetics physician and contributed to the manuscript. Helen Simpson was the patient's primary endocrine physician and contributed to the manuscript. Dr Simpson gives permission for this clinical report to be published.

\section{References}

1 Feldman BJ, Rosenthal SM, Vargas GA, Fenwick RG, Huang EA, Matsuda-Abedini M, Lustig RH, Mathias RS, Portale AA, Miller WL, et al. Nephrogenic syndrome of inappropriate antidiuresis. New England Journal of Medicine 2005352 1884-1890. (https://doi.org/10.1056/ NEJMoa042743)
2 Rosenthal W, Seibold A, Antaramian A, Lonergan M, Arthus MF, Hendy GN, Birnbaumer M \& Bichet DG. Molecular identification of the gene responsible for congenital nephrogenic diabetes insipidus. Nature 1992359 233-235. (https://doi.org/10.1038/359233a0)

3 Bockenhauer D, Penney MD, Hampton D, van't Hoff W, Gullett A, Sailesh S \& Bichet DG. A family with hyponatremia and the nephrogenic syndrome of inappropriate antidiuresis. American Journal of Kidney Diseases 201259 566-568. (https://doi. org/10.1053/j.ajkd.2011.09.026)

4 Brachet C, Vandergheynst F \& Heinrichs C. Nephrogenic syndrome of inappropriate antidiuresis in a female neonate: review of the clinical presentation in females. Hormone Research in Paediatrics 2015 84 65-67. (https://doi.org/10.1159/000381586)

5 Ranchin B, Boury-Jamot M, Blanchard G, Dubourg L, Hadj-Aïssa A, Morin D, Durroux T, Cochat P, Bricca G, Verbavatz JM, et al. Familial nephrogenic syndrome of inappropriate antidiuresis: dissociation between aquaporin-2 and vasopressin excretion. Journal of Clinical Endocrinology and Metabolism 201095 E37-E43. (https://doi. org/10.1210/jc.2009-2524)

6 Richards S, Aziz N, Bale S, Bick D, Das S, Gastier-Foster J, Grody WW, Hegde M, Lyon E, Spector E, et al. Standards and guidelines for the interpretation of sequence variants: a joint consensus recommendation of the American College of Medical Genetics and Genomics and the Association for molecular pathology. Genetics in Medicine 201517 405-424. (https://doi.org/10.1038/gim.2015.30)

7 Decaux G, Vandergheynst F, Bouko Y, Parma J, Vassart G \& Vilain C. Nephrogenic syndrome of inappropriate antidiuresis in adults: high phenotypic variability in men and women from a large pedigree. Journal of the American Society of Nephrology 200718 606-612. (https://doi.org/10.1681/ASN.2006090987)

8 Gupta S, Cheetham TD, Lambert HJ, Roberts C, Bourn D, Coulthard MG \& Ball SG. Thirst perception and arginine vasopressin production in a kindred with an activating mutation of the type 2 vasopressin receptor: the pathophysiology of nephrogenic syndrome of inappropriate antidiuresis. European Journal of Endocrinology 2009 161 503-508. (https://doi.org/10.1530/EJE-09-0246)

9 Erdélyi LS, Mann WA, Morris-Rosendahl DJ, Groß U, Nagel M, Várnai P, Balla A \& Hunyady L. Mutation in the V2 vasopressin receptor gene, AVPR2, causes nephrogenic syndrome of inappropriate diuresis. Kidney International 201588 1070-1078.

10 Powlson AS, Challis BG, Halsall DJ, Schoenmakers E \& Gurnell M. Nephrogenic syndrome of inappropriate antidiuresis secondary to an activating mutation in the arginine vasopressin receptor AVPR2. Clinical Endocrinology 201685 306-312. (https://doi.org/10.1111/ cen.13011)

Received in final form 12 December 2017

Accepted 16 January 2018 